\title{
Kinematics around the B335 protostar down to au scales ${ }^{\star}$
}

\author{
Per Bjerkeli ${ }^{1}$, Jon P. Ramsey ${ }^{2,4}$, Daniel Harsono ${ }^{3}$, Hannah Calcutt ${ }^{1}$, Lars E. Kristensen ${ }^{4}$, \\ Matthijs H. D. van der Wiel ${ }^{5}$, Jes K. Jørgensen ${ }^{4}$, Sébastien Muller ${ }^{1}$, and Magnus V. Persson ${ }^{1}$ \\ ${ }^{1}$ Department of Space, Earth, and Environment, Chalmers University of Technology, Onsala Space Observatory, \\ 43992 Onsala, Sweden \\ e-mail: per.bjerkeli@bjerkeli.se \\ 2 Department of Astronomy, University of Virginia, Charlottesville, VA 22904, USA \\ ${ }^{3}$ Leiden Observatory, Leiden University, PO Box 9513, 2300 RA Leiden, The Netherlands \\ ${ }^{4}$ Niels Bohr Institute and Centre for Star and Planet Formation, University of Copenhagen, Øster Voldgade 5-7, \\ 1350 Copenhagen K, Denmark \\ 5 ASTRON, the Netherlands Institute for Radio Astronomy, Oude Hoogeveensedijk 4, 7991 PD Dwingeloo, The Netherlands
}

Received 24 May 2019 / Accepted 6 September 2019

\begin{abstract}
Context. The relationship between outflow launching and the formation of accretion disks around young stellar objects is still not entirely understood, which is why spectrally and spatially resolved observations are needed. Recently, the Atacama Large Millimetre/sub-millimetre Array (ALMA) carried out long-baseline observations towards a handful of young sources, revealing connections between outflows and the inner regions of disks.

Aims. Here we aim to determine the small-scale kinematical and morphological properties of the outflow from the isolated protostar B335 for which no Keplerian disk has, so far, been observed on scales down to 10 au.

Methods. We used ALMA in its longest-baseline configuration to observe emission from CO isotopologues, $\mathrm{SiO}, \mathrm{SO}_{2}$, and $\mathrm{CH}_{3} \mathrm{OH}$. The proximity of B335 provides a resolution of $\sim 3$ au $\left(0.03^{\prime \prime}\right)$. We also combined our long-baseline data with archival observations to produce a high-fidelity image covering scales up to 700 au (7").

Results. ${ }^{12} \mathrm{CO}$ has an $\mathrm{X}$-shaped morphology with arms $\sim 50$ au in width that we associate with the walls of an outflow cavity, similar to what is observed on larger scales. Long-baseline continuum emission is confined to $<7$ au from the protostar, while short-baseline continuum emission follows the ${ }^{12} \mathrm{CO}$ outflow and cavity walls. Methanol is detected within $\sim 30$ au of the protostar. SiO is also detected in the vicinity of the protostar, but extended along the outflow.

Conclusions. The ${ }^{12} \mathrm{CO}$ outflow does not show any clear signs of rotation at distances $\gtrsim 30$ au from the protostar. SiO traces the protostellar jet on small scales, but without obvious rotation. $\mathrm{CH}_{3} \mathrm{OH}$ and $\mathrm{SO}_{2}$ trace a region $<16$ au in diameter, centred on the continuum peak, which is clearly rotating. Using episodic, high-velocity, ${ }^{12} \mathrm{CO}$ features, we estimate the launching radius of the outflow to be $<0.1$ au and dynamical timescales of the order of a few years.
\end{abstract}

Key words. stars: formation - stars: protostars - ISM: jets and outflows - accretion, accretion disks

\section{Introduction}

Protostellar outflows are arguably the most visibly prominent signature of ongoing star formation. Since their discovery almost four decades ago (Snell et al. 1980), a range of models have been proposed to explain how they are launched. The differences between these models mainly pertain to where acceleration of the outflow takes place (see, e.g. Frank et al. 2014, for a recent review), that is, close to the protostar (Shu et al. 1994) or throughout an extended region in the disk (Blandford \& Payne 1982; Pudritz \& Norman 1983; Lynden-Bell 1996). What all these models have in common, though, is the assertion that outflows are magnetically-powered.

Recently, we report on the first resolved images of an outflow launching region towards the Class I source TMC1A (Bjerkeli et al. 2016b), demonstrating that launching occurs from the disk at radii up to $\sim 25 \mathrm{au}$. Other observations carried out at high spatial resolution have since shown that launching can take place

\footnotetext{
* A copy of the reduced datacubes (FITS files) is available at the CDS via anonymous ftp to cdsarc.u-strasbg. fr $(130.79 .128 .5)$ or via http://cdsarc.u-strasbg.fr/viz-bin/cat/J/A+A/631/A64
}

out to large radii in the disk (e.g. Lee et al. 2018; Alves et al. 2017), but also close to the protostar itself (e.g. HH 212; Lee et al. 2018). These results underscore that outflow launching itself is still not entirely understood and, in particular, how it varies with the evolutionary stage of the disk.

To understand the launching of outflows, and specifically how angular momentum is transported throughout the system, one first needs to comprehend disk formation and its relation to outflow launching. Disk formation begins when a rotating molecular cloud core contracts under its own gravity (e.g. Shu et al. 1987). Due to the conservation of angular momentum during this inside-out collapse, infalling material that has too much angular momentum to fall directly onto the central protostar instead forms a rotating disk. The presence of a magnetic field during collapse, meanwhile, provides a means to efficiently transport angular momentum, which can strongly affect disk formation. The initial conditions for the disk structure, as well as its early evolution, are set during this deeply embedded phase of star formation, that is, the Class 0 stage (Andre et al. 2000). In this phase, collapse proceeds inside-out, and infalling motion initially dominates over rotational motions. Over time, 
the infalling material begins to accumulate in a disk that slowly settles into nearly Keplerian rotation. The details of when and how the disk becomes Keplerian, however, is still under debate (e.g. Li et al. 2014a; Wurster \& Li 2018).

In the presence of a large-scale magnetic field, the interaction between the rotation of the system (disk and star) and the magnetic field promotes the launching of an outflow perpendicular to the disk. This outflow not only carries angular momentum extracted from the disk, but also mass. Numerical studies meanwhile show that outflows can already form during first-core formation (Tomida et al. 2013). Numerous studies (e.g. Li et al. 2011; Machida et al. 2014; Tomida et al. 2015) show that magnetic fields can also slow down infall and prevent the formation of a Keplerian disk (the so-called "magnetic braking catastrophe") during the earliest stages. In this scenario, in order to form disks, one must introduce non-ideal magnetic effects (e.g. Zhao et al. 2016) and/or turbulence (e.g. Seifried et al. 2013; Li et al. 2014b).

The advent of the Atacama Large Millimetre/sub-millimetre Array (ALMA) is revolutionising our understanding of star and disk formation. To date, ALMA observations reveal Keplerian disks towards several very young sources (e.g. Tobin et al. 2012; Murillo et al. 2013; Lindberg et al. 2014; Ohashi et al. 2014). However, ALMA has also revealed counter examples where a Keplerian disk has not been detected yet. One such example is the Class 0 protostar B335.

B335 is an isolated dense globule associated with the infrared identified protostar "IRAS 19347+0727" at a distance of approximately 100 pc (90-120 pc; Olofsson \& Olofsson 2009). Although recent studies (Evans et al. 2015; Yen et al. 2015) reveal that matter infalls towards the centre of B335, these studies do not resolve any Keplerian components on scales greater than $\sim 10$ au in size. From the standard paradigm of protostellar disk formation theory, the absence of a rotationally supported disk on these scales suggests that the B335 system is either very young, and/or that it is subject to strong magnetic braking (e.g. Yen et al. 2015).

A young age for B335 is also supported by recent single-dish observations of its outflow. Observations of the molecular component (Y1ldiz et al. 2015) imply that dynamical time-scales for the $\mathrm{CO}$ emitting gas are of the order of $10^{4} \mathrm{yr}$. This suggests that the B335 system is extremely young, and therefore, it is an ideal target to study early and ongoing star and disk formation. Furthermore, B335 is known to power a fast protostellar jet and associated Herbig-Haro (HH) objects (Gålfalk \& Olofsson 2007; Reipurth et al. 1992). Based on the proper motions of the $\mathrm{HH}$ objects, the estimated dynamical time-scale is only a few hundred years.

Several saturated complex organic molecules indicative of hot corino chemistry (for example, $\mathrm{CH}_{3} \mathrm{CHO}, \mathrm{HCOOCH}_{3}$ and $\mathrm{NH}_{2} \mathrm{CHO}$ ) have been detected on small scales towards $\mathrm{B} 335$ (Imai et al. 2016). More recently, Imai et al. (2019) observed rotation in $\mathrm{CH}_{3} \mathrm{OH}$ and $\mathrm{HCOOH}$ towards $\mathrm{B} 335$ on scales of $\sim 0.2^{\prime \prime}(\sim 20 \mathrm{au}) . \mathrm{CH}_{3} \mathrm{OH}$ is a molecule of key interest when it comes to the formation of organics (Herbst \& van Dishoeck 2009). Understanding the link between emerging disks and such complex species therefore remains very important for understanding the initial conditions of protoplanetary disk chemistry. While $\mathrm{CH}_{3} \mathrm{OH}$ is often seen to be associated with high column density material in protostellar envelopes, as well as shocks in outflows, to date, it has only been detected in the disks around two sources, TW Hya and V883 Ori (Walsh et al. 2016; van 't Hoff et al. 2018), both of which are significantly older than B335.
The morphology of the molecular component (e.g. Yen et al. 2010) traced by CO (2-1), meanwhile, reveals an X-shaped structure with an opening angle of the order of $\sim 45^{\circ}$. The outflow is nearly in the plane of the sky with an inclination angle that is between $3^{\circ}$ and $10^{\circ}$ (Hirano et al. 1988; Stutz et al. 2008) ${ }^{1}$.

The age, the orientation, and the proximity of B335 make it an excellent target for long-baseline observations with ALMA. In its most extended configuration, ALMA can attain an angular resolution of $\sim 0.02^{\prime \prime}$, providing a maximum linear resolution of $\sim 2$ au for the distance of B335. Such a high spatial resolution enables detailed studies of both the innermost protostellar region and the outflow launching region.

\section{Observations}

\subsection{New long-baseline observations}

B335 was observed with ALMA between October 21 and 29, 2017 (five execution blocks) as part of the Cycle 5 programme 2017.1.00288.S. Observations were carried out in Band 6 and cover five spectral windows (SPWs). Three of them were centred on $\mathrm{CO}$ isotopologues, viz., the $J=2-1$ transitions of ${ }^{12} \mathrm{CO}$, ${ }^{13} \mathrm{CO}$, and $\mathrm{C}^{18} \mathrm{O}$ at $230.5 \mathrm{GHz}, 220.4 \mathrm{GHz}$, and $219.6 \mathrm{GHz}$, respectively. The $\mathrm{SiO}(5-4)$ transition was covered in the fourth SPW centred at $217.1 \mathrm{GHz}$, while the fifth SPW targeted the continuum at $233.0 \mathrm{GHz}$. Between 45 and 51 antennas of the $12 \mathrm{~m}$ array were used. Baselines were in the range 41-16 $196 \mathrm{~m}$, yielding a spatial resolution of $0.03^{\prime \prime}$ and a largest recoverable scale corresponding to $\sim 0.3^{\prime \prime 2}$. For the adopted distance of B335, this implies a linear resolution of $\sim 3$ au, but also that these observations are not sensitive to structures with angular scales larger than $\sim 30$ au, such as, the foreground envelope emission and large-scale outflow emission. The spectral resolution was set to $122 \mathrm{kHz}$ for the ${ }^{12} \mathrm{CO} \mathrm{SPW}$, and $61 \mathrm{kHz}$ for ${ }^{13} \mathrm{CO}$ and $\mathrm{C}^{18} \mathrm{O}$, while the total bandwidth in each SPW was $120 \mathrm{MHz}$. The continuum was observed at a spectral resolution of $31.25 \mathrm{MHz}$, while the $217.1 \mathrm{GHz}$ SPW centred on $\mathrm{SiO}$ had a bandwidth of $937 \mathrm{MHz}$ and a spectral resolution $488 \mathrm{kHz}$. Over the course of the observations, the precipitable water vapour (PWV) ranged from 0.5 to $1.5 \mathrm{~mm}$. The phase centre of the observations were $\alpha_{2000}=19^{\mathrm{h}} 37^{\mathrm{m}} 00^{\mathrm{s}} .890, \delta_{2000}=+07^{\circ} 34^{\prime} 09.60^{\prime \prime} . \mathrm{J} 2000-1748$, J2134-0153 and J2148+0657 were used as bandpass calibrators, while J1938+0448 was used to calibrate the phase. The flux calibration was done using J2000-1748, J2134-0153, and $\mathbf{J} 2148+0657$. We estimate a flux accuracy of $\sim 10 \%$. Data reduction and imaging were carried out in CASA v5.1.1 (McMullin et al. 2007). The continuum was subtracted in the $u v$ domain using only line-free channels. Calibrated visibilities were then transferred into the image domain using the CLEAN algorithm with Briggs weighting and a robust parameter of 0.5 . All spectral line maps were imaged at the native spectral resolution.

\subsection{Combining with archived data}

The largest recoverable scales in our long-baseline data are much smaller than the known extent of the $\mathrm{CO}(2-1)$ emission in B335 (e.g. Yen et al. 2010). As such, short-spacing data is needed to capture any large-scale outflow emission filtered out by our long-baseline observations and make the connection to our

\footnotetext{
1 Herein, we define an inclination angle of $0^{\circ}$ to coincide with features that lie in the plane of the sky.

2 ALMA Technical Handbook, https://almascience.eso.org
} 
Table 1. Summary of all ALMA data sets employed in this study.

\begin{tabular}{lllllllll}
\hline \hline Project & Date & $N_{\text {ant }}$ & $\begin{array}{l}t_{\text {on }} \\
(\mathrm{s})\end{array}$ & $\begin{array}{l}\text { PWV } \\
(\mathrm{mm})\end{array}$ & $\begin{array}{l}B_{\min } \\
(\mathrm{m})\end{array}$ & $\begin{array}{l}B_{\max } \\
(\mathrm{m})\end{array}$ & DR & $\begin{array}{l}\Delta \text { (RA, Dec }) \\
(\mathrm{mas})\end{array}$ \\
\hline 2013.1.00879.S & 02-Sep-2014 & 34 & 1463 & 1.0 & 32 & 1052 & 69 & $-80,95$ \\
2016.1.01552.S & 21-Nov-2016 & 43 & 1851 & 2.1 & 15 & 704 & 67 & $32,-65$ \\
& 19-Mar-2017 & 40 & 685 & 1.6 & 15 & 287 & 34 & $-11,24$ \\
2017.1.00288.S & 08-Oct-2017 & 51 & 2268 & 0.5 & 41 & 16196 & 99 & $-19,-1$ \\
& 21-Oct-2017 & 49 & 2268 & 1.4 & 41 & 16196 & 64 & $-15,0$ \\
& 22-Oct-2017 & 46 & 2268 & 1.3 & 41 & 16196 & 62 & $-13,0$ \\
& 27-Oct-2017 & 47 & 2271 & 0.7 & 135 & 14851 & 94 & $-14,-3$ \\
& 29-Oct-2017 & 45 & 2268 & 1.5 & 113 & 13894 & 37 & $-15,-2$ \\
\hline
\end{tabular}

Notes. $N_{\text {ant }}=$ number of antennas in the array; $t_{\mathrm{on}}=$ on-source integration time; PWV = precipitable water vapour; $B_{\min }=$ shortest projected baseline; $B_{\max }=$ longest projected baseline; $\mathrm{DR}=$ dynamic range of the continuum data; $\Delta(\mathrm{RA}, \mathrm{Dec})=$ astrometry offsets for the continuum peak, relative to the phase centre set at $\alpha_{2000}=19^{\mathrm{h}} 37^{\mathrm{m}} 00^{\mathrm{s}} 890, \delta_{2000}=+07^{\circ} 34^{\prime} 09.60^{\prime \prime}$.

higher-resolution data. Our goal is, therefore, to produce a highas-possible fidelity, and simultaneously high-angular resolution, image of the emission in B335, covering all relevant scales.

To accomplish this, we combined our long-baseline data with two publicly-available ALMA data sets towards B335 from 2014 (2013.1.00879.S) and 2016/2017 (2016.1.01552.S). The spectral setup for both archival data sets covered the ${ }^{12} \mathrm{CO}(2-1)$ line, and calibration was performed using CASA v4.3.1 and v4.7.0, respectively. Care is needed when combining data taken with different array configurations (see Table 1), including w.r.t. the flux accuracy, relative astrometry, and handling of the weights in each individual data set. Data combination was carried out in the following manner: first, we cleaned the continuum emission from different data sets individually, using the Högbom deconvolution algorithm (Högbom 1974), reprojecting all data to the same phase centre. We used a 2D-Gaussian fit to the peak position of the compact continuum emission in each data set to constrain their relative astrometry. After performing the 2D Gaussian fits and a visual inspection, relatively small offsets (although in a few cases slightly larger than the final beam size) between the different data sets were found, as indicated in Table 1. We also checked the dynamic range of each data set by taking the ratio of the continuum peak to the noise level in the residuals of the cleaned product. The weights of each data set were normalised by dividing them by their average (that is, all data sets received the same average weight level). We found that the dynamic ranges were consistent within a factor of three, and hence, we decided to not modify further the relative weightings. During the combination, we manually flagged (and consequently never used) some of the data because it was of bad quality.

Next, we deconvolved the combined data set using the CASA tclean procedure. Briggs weighting was used with the robust parameter set to 0.5 . In all cases, the spectral resolution was sufficiently high to resolve the ${ }^{12} \mathrm{CO}(2-1)$ line with $0.25 \mathrm{~km} \mathrm{~s}^{-1}$. The angular resolution of the 2013 project is $\sim 0.3^{\prime \prime}$, while the $2016 / 2017$ project was observed in two different configurations corresponding to spatial resolutions of $\sim 0.7^{\prime \prime}$ and $\sim 1.5^{\prime \prime}$, respectively. By combining these data sets, we were able to recover emission on scales from $\sim 0.03^{\prime \prime}$ up to $\sim 7^{\prime \prime}$ (see Fig. 1 for the different $u, v$ coverages). The cleaning threshold for the continuum was set to $0.05 \mathrm{mJy}^{-1}$ beam $^{-1}$ and the threshold for the lines was set to $3 \mathrm{mJy}$ beam $^{-1}$. Due to the nature of the combined data set, we used the so-called multiscale deconvolution algorithm for the line emission. The input scales used were $0,8,23,68,203$, and 608 pixels, while the pixel scale was set to $0.01^{\prime \prime}$. The size of the
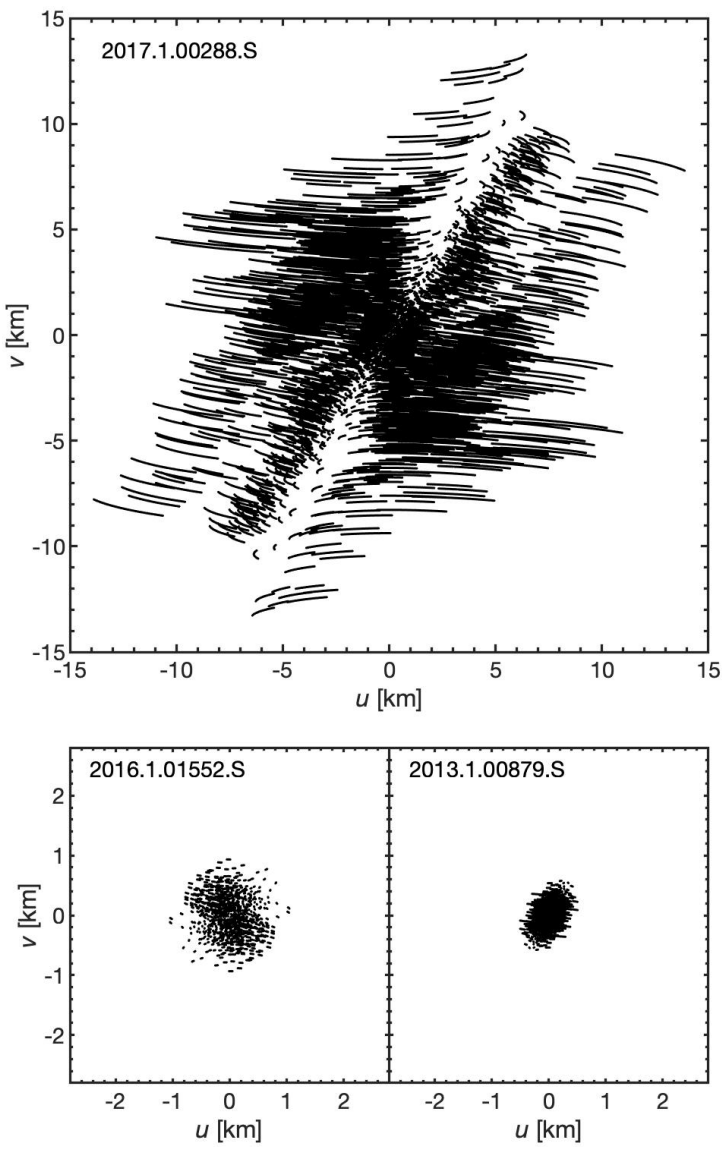

Fig. 1. $u, v$ coverage for the three different ALMA projects. We note that the scale changes between panels.

cleaned image is 4096 by 4096 pixels. For the line emission, we performed test cleans on a few channels close to the systemic velocity to confirm that changing the cleaning scales does not significantly affect the results. To ensure the combined image was consistent with the individual data sets, we also cleaned the different ${ }^{12} \mathrm{CO}$ data sets with the Högbom deconvolution algorithm and compared the results to the multiscale cleaned image. Indeed, convolving the final combined data product with 2D Gaussians (with FWHM corresponding to the angular resolution of each data set) reproduces the overall morphology of the emission observed in projects 2013.1.00879 and 2016.1.01552. 


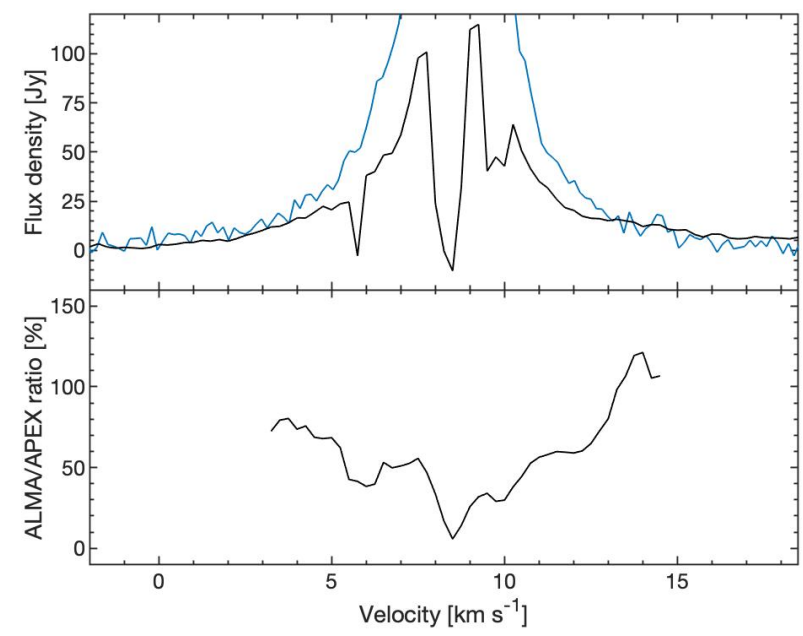

Fig. 2. Upper panel: combined multiscale ${ }^{12} \mathrm{CO}$ spectrum (black), integrated over the field of view, as compared to the single-dish APEX spectrum (blue) observed towards the position of B335. Lower panel: moving average (using $1.0 \mathrm{~km} \mathrm{~s}^{-1}$ bins) of the ALMA-to-APEX ratio of fluxes in regions where the APEX $S / N>3 \sigma$.

It is worth noting that the multiscale cleaning of the combined data set required significant computing resources. For the combined ${ }^{12} \mathrm{CO}$ image presented in Fig. 4, one dedicated node (256 GB RAM) of the C3SE Hebbe cluster ${ }^{3}$ was used for 800 wall-clock hours. Even after that amount of time, a few channels were not properly cleaned (that is, they had not reached the desired threshold of $3 \mathrm{mJy} / \mathrm{beam})$.

To check the amount of ${ }^{12} \mathrm{CO}(2-1)$ flux recovered in these observations, we compared the final combined image with single dish data acquired with the Atacama Pathfinder Experiment (APEX) telescope (Project: O-087.F-9314A, PI: M. V. Persson). Figure 2 demonstrates that more than $50 \%$ of the emission observed with APEX is recovered in the line wings ( $>2 \mathrm{~km} \mathrm{~s}^{-1}$ from systemic velocity) of the ALMA data. Also evident from this figure are the channels where cleaning was not carried out deeply enough, that is, one channel at approximately $+6.0 \mathrm{~km} \mathrm{~s}^{-1}$ and a few channels at +9.5 to $+10.0 \mathrm{~km} \mathrm{~s}^{-1}$.

For the analysis that follows, we use the long-baseline data when analysing the ${ }^{13} \mathrm{CO}, \mathrm{C}^{18} \mathrm{O}, \mathrm{SiO}, \mathrm{SO}_{2}$, and $\mathrm{CH}_{3} \mathrm{OH}$ emission, while we use primarily the combined dataset when analysing the ${ }^{12} \mathrm{CO}$ and continuum emission. The analysis was performed with MATLAB.

\section{Results}

\subsection{Continuum and carbon monoxide}

In the long-baseline data, continuum emission at $1.3 \mathrm{~mm}$ was detected with a peak flux of $4.8 \mathrm{mJy} \mathrm{beam}^{-1}\left(\sigma \simeq 2 \times 10^{-5} \mathrm{Jy}\right)$ towards $\alpha_{2000}=19^{\mathrm{h}} 37^{\mathrm{m}} 00^{\mathrm{s}} 900, \delta_{2000}=+07^{\circ} 34^{\prime} 09.52^{\prime \prime} \quad($ see Fig. 6). The emission is approximately Gaussian with a $\sim 5$ au extension to the north-west. The full width half-maximum (FWHM) of the continuum was estimated using 2D Gaussian fitting in the image plane and found to be $\sim 7$ au, in other words, slightly extended relative to the size of the synthesised beam. We also imaged the continuum using the combined data set. While the continuum is only detected towards the central position in

3 Chalmers Centre for Computational Science and Engineering: https://www.c3se. chalmers. se

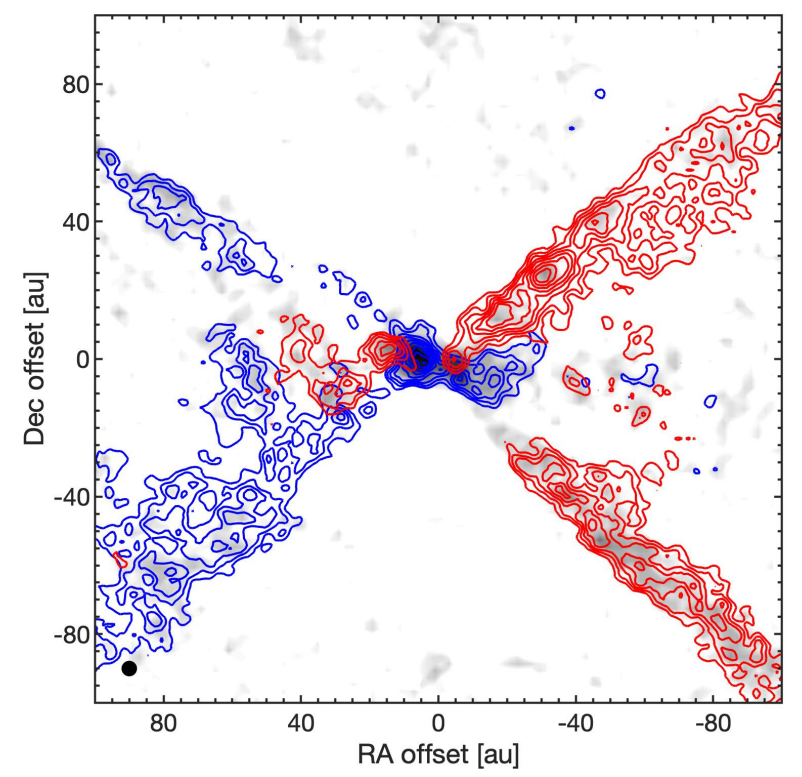

Fig. 3. Combined ${ }^{12} \mathrm{CO}(2-1)$ emission convolved with a $2 \mathrm{D}$ Gaussian to a resolution of 4 au and integrated from 2.0 to $10.0 \mathrm{~km} \mathrm{~s}^{-1}$ relative to the source velocity, $8.3 \mathrm{~km} \mathrm{~s}^{-1}$ w.r.t. $v_{\text {LSR }}$. Contours are from $3 \sigma$ in steps of $1 \sigma$, where $\sigma=3.3 \mathrm{mJy}^{-1} \mathrm{beam}^{-1} \mathrm{~km} \mathrm{~s}^{-1}$. Greyscale shows the corresponding map when using the long-baseline 2017.1.00288 data only. The convolved beam is shown in the lower left corner of the map.

the long-baseline observations, the combined data set shows extended emission on much larger scales (Figs. 4 and A.1).

The systemic velocity of B335 has previously been estimated to be in the 8.3-8.5 $\mathrm{km} \mathrm{s}^{-1}$ range (Evans et al. 2005; Jørgensen et al. 2007; Yen et al. 2011; Mottram et al. 2014). In the analysis presented in this paper, we adopt a source velocity of $8.3 \mathrm{~km} \mathrm{~s}^{-1}$ based on rare, isotopic line emission (Evans et al. 2005). That is roughly consistent with what we find from the $\mathrm{CO}$ isotopologue observations presented here: by extracting mean spectra over a 50 au diameter circular region centred on the continuum peak, we find the systemic velocity to be $\sim 8.5 \mathrm{~km} \mathrm{~s}^{-1}$ w.r.t. $v_{\mathrm{LSR}}$ in all three $\mathrm{CO}$ isotopologues, independent of whether we use the combined data set or the long-baseline data only.

Previous observations (e.g. Yen et al. 2010), have shown that the $\mathrm{CO}(2-1)$ emission is prominent along the outflow cavity walls where envelope gas is expected to be entrained. That is consistent with what we find here. In our long-baseline data, ${ }^{12} \mathrm{CO}$ emission is detected predominantly towards the outflow emanating from B335, where the peak flux is $\sim 12 \mathrm{mJy} \mathrm{beam}^{-1}$ at a signal-to-noise ratio $(\mathrm{S} / \mathrm{N})$ of $\sim 7$. From comparison with APEX single-dish data, however, it is obvious that a significant fraction of the emission originates on scales greater than the MRS of the long-baseline observations, viz., $0.3^{\prime \prime}$ (recovered flux is less than $20 \%$ in the line wings). Therefore, for the remainder of the ${ }^{12} \mathrm{CO}$ analysis presented in this paper we use the multiscale cleaned image. Figure 3 presents the integrated ${ }^{12} \mathrm{CO}$ emission from the combined data set (contours) overlaid on top of the long-baseline only data (greyscale). A similar, but larger-scale and slightly smoothed $\left(\sim 0.1^{\prime \prime}\right)$ map is shown in Fig. 4, where spectra towards a few selected positions are also presented.

Maximum observed velocities of the main line component are $\sim 10 \mathrm{~km} \mathrm{~s}^{-1}$ w.r.t. the systemic velocity, consistent with previous single dish ${ }^{12} \mathrm{CO}(3-2)$ observations of B335 (Y1ldiz et al. 2015). In addition, however, we observe two additional emission peaks at $-20 \mathrm{~km} \mathrm{~s}^{-1}$ and at $+40 \mathrm{~km} \mathrm{~s}^{-1}$ at positions close to the central source (see black point/spectra in Fig. 4) and in 

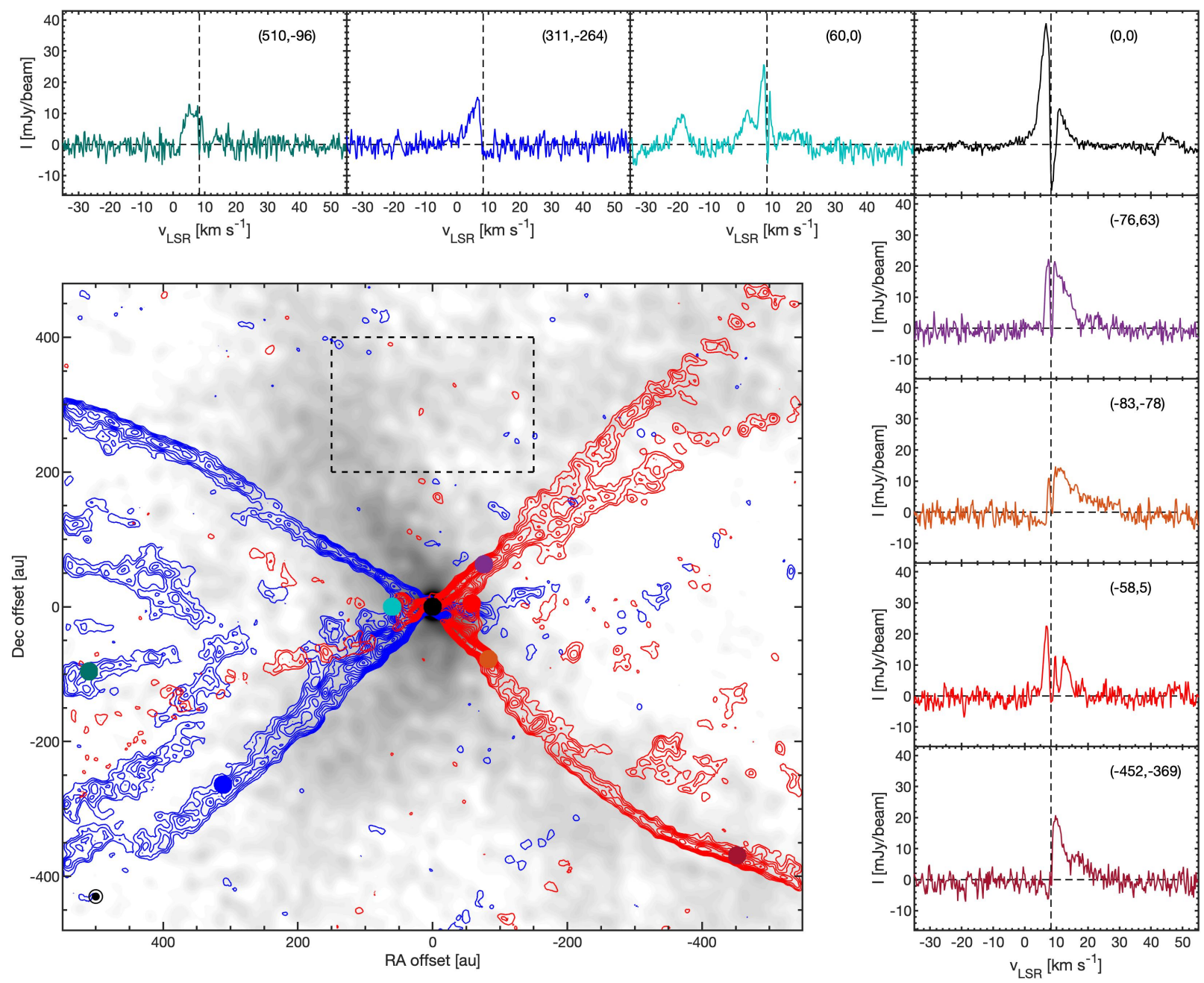

Fig. 4. Moment 0 map of the ${ }^{12} \mathrm{CO}$ emission (contours from $3 \sigma$ in steps of $1 \sigma$, where $\sigma=5.6 \mathrm{mJy}$ beam $^{-1}$ ), overlaid on the continuum (greyscale from 0 to $25 \%$ of maximum), in the combined data set. The ${ }^{12} \mathrm{CO}$ image was convolved with a $2 \mathrm{D}$ Gaussian to 10 au resolution to enhance the $\mathrm{S} / \mathrm{N}$ in the map, while the continuum was convolved with a $2 \mathrm{D}$ Gaussian to 20 au resolution. The emission is integrated from $2.0-6.0 \mathrm{~km} \mathrm{~s}{ }^{-1}$ with respect to the source velocity, $8.3 \mathrm{~km} \mathrm{~s}^{-1}$ w.r.t. $v_{\text {LSR }}$. The dashed box denotes where the line rms was calculated using the line free channels. Selected mean spectra averaged over circular regions (10 au in radius) are indicated by the coloured points and the corresponding coloured spectral profiles. The convolved beams are shown in the lower left corner of the map. For clarity, a zoomed-out version of this figure with only the continuum data is presented in Fig. A.1.

the outflow at a distance of $\sim 60$ au from the central source (cyan point/spectra in Fig. 4), respectively. We interpret these peaks as higher velocity components potentially associated with the known high-velocity protostellar jet (Gålfalk \& Olofsson 2007). It should be noted, however, that the $\mathrm{SiO}(5-4)$ emission (Sect. 3.2) does not extend out to the positions where the high-velocity features are detected, but this may simply be due to insufficient sensitivity, and it is therefore difficult to confirm the origin of the high velocity ${ }^{12} \mathrm{CO}$ peaks. The observed velocity shifts of these components are, in fact, similar to the velocity extent of the $\mathrm{H}_{2} \mathrm{O}\left(1_{10}-1_{01}\right)$ line observed with Herschel-HIFI towards the central position (Kristensen et al. 2012; Mottram et al. 2014), but a more detailed comparison with these observations is hindered by the low $\mathrm{S} / \mathrm{N}$ of the water data.

The ${ }^{12} \mathrm{CO}$ emission shows a pronounced X-shaped morphology (Fig. 4) that first becomes apparent in our data set on scales $\sim 15 \mathrm{au}$, and extends well beyond the MRS of even the combined data to $\gtrsim 2000$ au (e.g. Yen et al. 2010). A clear red-blue asymmetry where blueshifted emission is detected in the eastern outflow component, and redshifted emission is detected in the western outflow component, is observed. It is also evident that the emission is dominated by the cavity walls plus a moderate amount of emission predominantly in the blueshifted outflow between the cavity walls. We note the presence of a prominent secondary arc in the blueshifted component, offset $\gtrsim 300$ au from the continuum peak, but, at present, its nature remains unclear. There is also a small amount of redshifted emission present on the blueshifted side of the outflow (and vice versa on the redshifted side). In the integrated emission map (Fig. 3), one can also see a partial band of emission, roughly 50 au from the protostar, seemingly connecting the two blueshifted outflow cavity walls together. Traces of a counterpart in the redshifted outflow are also visible at the $3-4 \sigma$ level. These features are indeed spatially coincident with the aforementioned high velocity components. We return to these features and their interpretation in Sect. 4.

In the long-baseline data set, ${ }^{13} \mathrm{CO}$ is detected only in close proximity to the central continuum peak. No significant ${ }^{13} \mathrm{CO}$ emission is detected towards the outflow cavity walls traced by ${ }^{12} \mathrm{CO}$. The maximum observed velocities of ${ }^{13} \mathrm{CO}$, however, coincide with the maximum velocities observed in ${ }^{12} \mathrm{CO}$ close to 


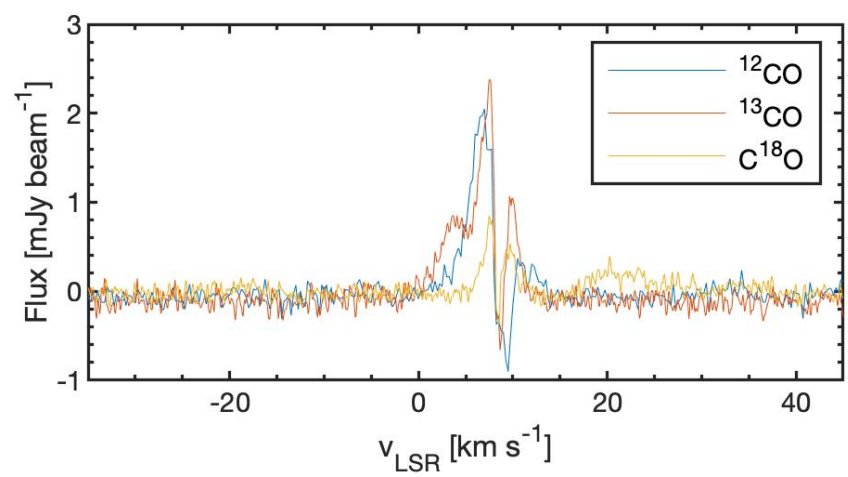

Fig. 5. Mean ${ }^{12} \mathrm{CO},{ }^{13} \mathrm{CO}$, and $\mathrm{C}^{18} \mathrm{O}$ spectra extracted from a circular region of diameter 50 au centred on the protostar. Data is from the longbaseline observations only.

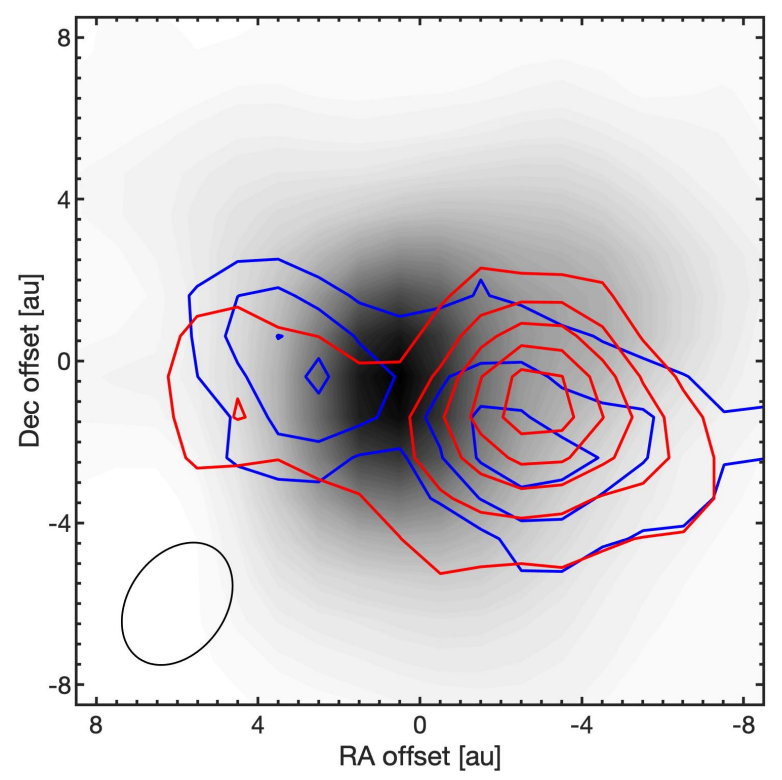

Fig. 6. Integrated blue- and redshifted $\mathrm{SiO}(5-4)$ emission $( \pm 1$ to \pm 10 $\mathrm{km} \mathrm{s}^{-1}$ w.r.t. systemic velocity; contours) overlaid on the $1.3 \mathrm{~mm}$ continuum emission (greyscale). Contours are from $3 \sigma$ in steps of $1 \sigma$, where $\sigma=2.1 \mathrm{mJy}$ beam ${ }^{-1} \mathrm{~km} \mathrm{~s}^{-1}$. The synthesized beam is shown in the lower left corner of the map.

the central position. $\mathrm{C}^{18} \mathrm{O}$ was also covered in our observations, but was only detected after degrading the spatial resolution, and even then only at low S/N. No conclusions can be drawn with regard to its spatial distribution and consequently we have chosen not to discuss the data further here. For completeness, and to aid comparison, Fig. 5, shows the spectra of the three $\mathrm{CO}$ isotopologues towards a 50 au sized region centred on the continuum peak. We note that all three line profiles show red-blue asymmetries indicating infall, and the line ratios suggest that the emission is optically thick in ${ }^{12} \mathrm{CO}$ and ${ }^{13} \mathrm{CO}$.

\section{2. $\mathrm{SiO}, \mathrm{CH}_{3} \mathrm{OH}$, and $\mathrm{SO}_{2}$}

Along with the observation of the $\mathrm{CO}$ isotopologues, one of the SPWs in our long-baseline data set covered the frequency range 216.6-217.6 GHz centred on the $\mathrm{SiO}(5-4)$ emission line. Figure 6 shows our detection of $\mathrm{SiO}$, overlaid on the continuum emission. $\mathrm{SiO}$ was only recently detected in the vicinity of the B335 protostar (Imai et al. 2019). Those observations were acquired at a spatial resolution of $0.1^{\prime \prime}$, and no extension in the

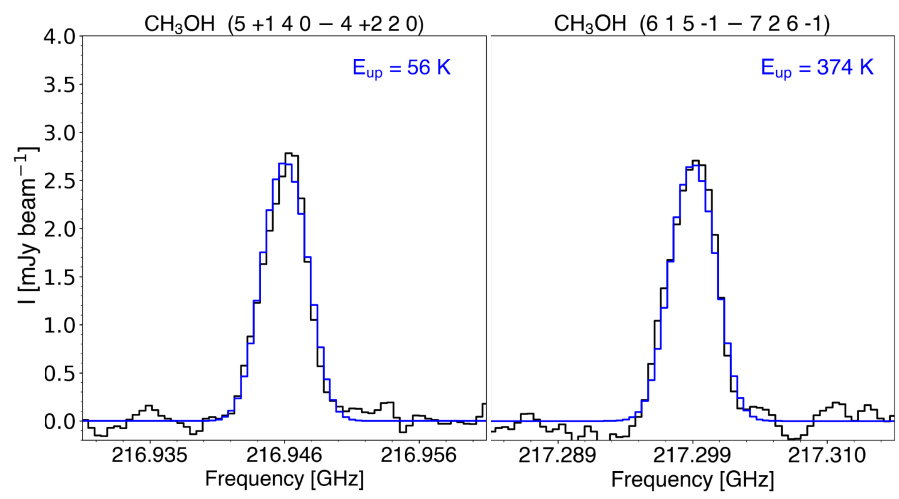

Fig. 7. $\mathrm{CH}_{3} \mathrm{OH} 5_{-1,4}-4_{-2,3} \mathrm{E}\left(\mathrm{v}_{t}=0\right)$ and $\mathrm{CH}_{3} \mathrm{OH} 6_{1,5}-7_{2,5} \mathrm{~A}\left(\mathrm{v}_{t}=1\right)$ lines of $\mathrm{CH}_{3} \mathrm{OH}$ detected towards $\mathrm{B} 335$ (black line) with an LTE spectral model overlaid in blue.

outflow direction could be resolved. Imai et al. (2019) suggests that $\mathrm{SiO}$ could be tracing the launching point of the outflow or the accretion shock of infalling material. In the higher resolution observations of this paper, however, we spatially resolve the $\mathrm{SiO}$ emission and find that it is elongated along the outflow direction on both sides of the protostar. Since, theoretically, magnetically-powered jets are expected to rotate (e.g. Blandford \& Payne 1982), we searched for, but could not find, any signs of rotation in $\mathrm{SiO}$ along the expected jet axis. This is most likely because the $\mathrm{SiO}$ emission is not well-resolved in the north-south direction (the FWHM of the SiO emission in the direction perpendicular to the outflow axis is comparable to the size of the beam).

Serendipitously, in the same SPW, we detected $\mathrm{CH}_{3} \mathrm{OH}$ $5_{-1,4}-4_{-2,3} \mathrm{E}\left(\mathrm{v}_{t}=0\right)$ at $216.945 \mathrm{GHz}\left(E_{\text {up }}=56 \mathrm{~K}\right), \mathrm{CH}_{3} \mathrm{OH} 6_{1,5}-$ $7,5 \mathrm{~A}\left(\mathrm{v}_{t}=1\right)$ at $217.299 \mathrm{GHz}\left(E_{\mathrm{up}}=374 \mathrm{~K}\right)$, and $\mathrm{SO}_{2} 22(2,20)$ $22(1,21)$ at $216.643 \mathrm{GHz}$ within a circular region of radius $\simeq 15 \mathrm{au}$ centred on the protostar. Although, the emission from all of the aforementioned species suffers absorption towards the protostellar position, they are otherwise well represented by Gaussian 2D fits. Moment 0 maps and position-velocity (PV) diagrams of these transitions are found in Figs. 8 and A.2.

\subsection{Deriving the excitation conditions from $\mathrm{CH}_{3} \mathrm{OH}$}

Four lines of $\mathrm{CH}_{3} \mathrm{OH}$ fall within the frequency range of our long-baseline observations. Two of these lines, $\mathrm{CH}_{3} \mathrm{OH} 5_{-1,4}$ $4_{-2,3} \mathrm{E}\left(\mathrm{v}_{t}=0\right)$ at $216.945 \mathrm{GHz}$ and $\mathrm{CH}_{3} \mathrm{OH} 6_{1,5}-7_{2,5} \mathrm{~A}\left(\mathrm{v}_{t}=1\right)$ at $217.299 \mathrm{GHz}$ are detected at a $\sim 20 \sigma$ level. The $6_{-3,4}-7_{-1,6} \mathrm{E}$ $\left(\mathrm{v}_{t}=0\right)$ and $16_{-1,15}-15_{-3,13} \mathrm{E}\left(\mathrm{v}_{t}=0\right)$ lines are not detected. By combining the upper energy levels and line strengths of these four lines, we can strongly constrain the excitation temperature and column density of the $\mathrm{CH}_{3} \mathrm{OH}$ emitting region with the spectral modelling code CASSIS ${ }^{4}$. Assuming local thermodynamic excitation (LTE) and optically thin emission, we ran a grid of models covering a range of column densities and excitation temperatures. The source size was taken to be $0.15^{\prime \prime}$ based on a $2 \mathrm{D}$ Gaussian fit to the $\mathrm{CH}_{3} \mathrm{OH}$ emission (see Sect. 4.2). The spectroscopic information was taken from the $\mathrm{CDMS}^{5}$ catalogue (Müller et al. 2001, 2005; Endres et al. 2016) entry for $\mathrm{CH}_{3} \mathrm{OH}$. Figure 7 shows the observed spectra (in black) extracted from a $0.15^{\prime \prime}$ region centred on the emission peak of B335, as

\footnotetext{
4 CASSIS has been developed by IRAP-UPS/CNRS: http:// cassis.irap.omp.eu/

5 The Cologne Database for Molecular Spectroscopy: https:// cdms.astro.uni-koeln.de/
} 

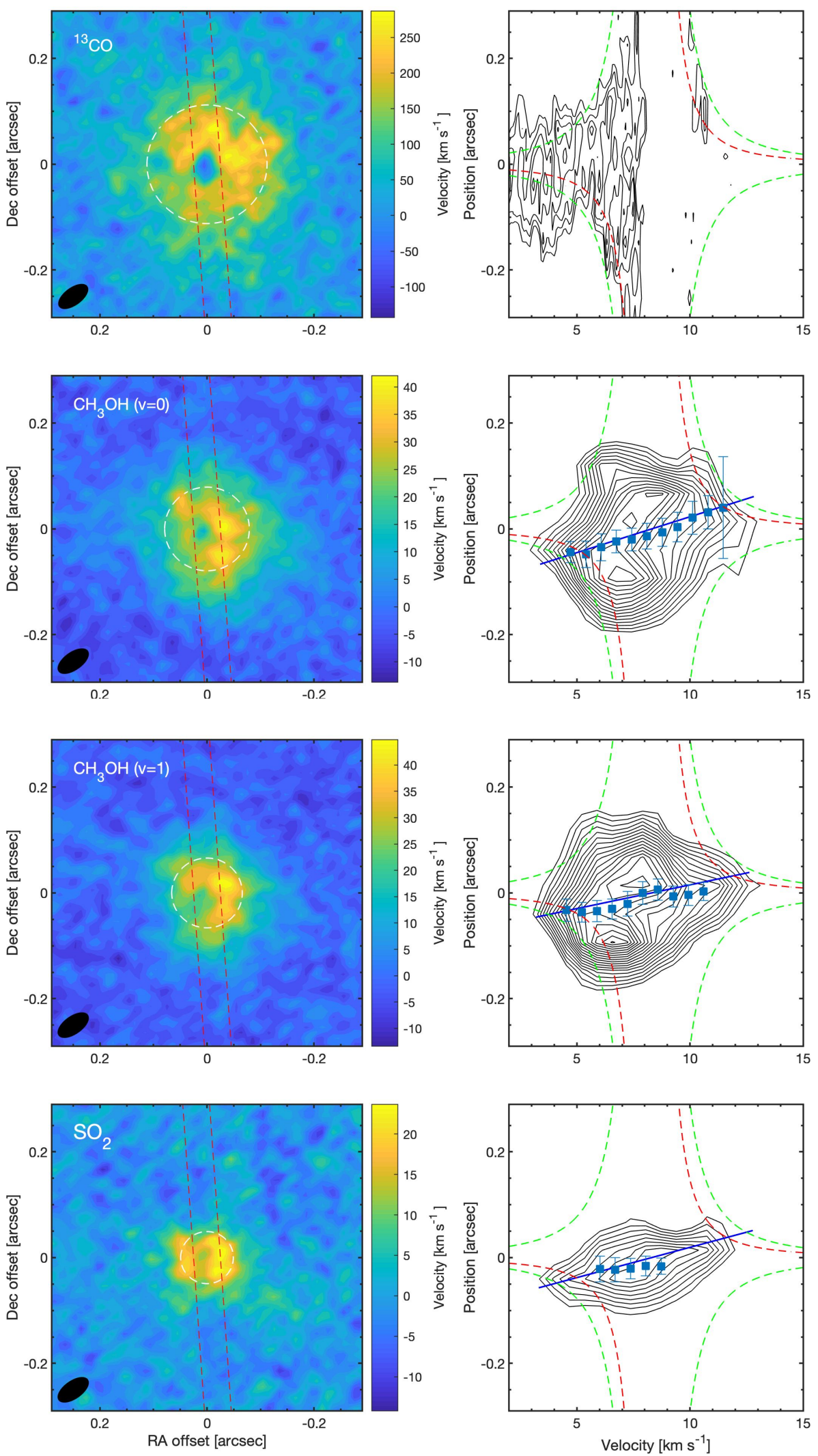

Fig. 8. ${ }^{13} \mathrm{CO}, \mathrm{CH}_{3} \mathrm{OH}(v=0,1)$, and $\mathrm{SO}_{2}$ integrated emission and PV diagrams. Left panels: emission integrated over $10 \mathrm{~km} \mathrm{~s}^{-1}$ centred on the systemic velocity. White dashed circles denote the FWHM of 2D Gaussian fits to the emission, while the red dashed lines indicate the direction and width of the PV cuts. The same PV cut is used in all panels. Synthesized beams are shown in the lower left corners. Right panels: resulting PV diagrams. Blue dots denote the location of emission peaks at a particular velocity (only velocity channels where the coefficient of determination, $\mathrm{R}^{2}$, is larger than 0.3 are included). A linear fit to the peak emission velocity profile is represented by the blue line. Pure free-fall and pure Keplerian rotation velocities towards/around a $0.05 M_{\odot}$ protostar are indicated in the right panels by the green and red dashed lines, respectively. well as the best-fit spectral model (in blue). In order to reproduce the detected lines, and not produce emission for the two non-detected lines, a $\mathrm{CH}_{3} \mathrm{OH}$ column density of $6.8 \pm 0.1 \times$ $10^{18} \mathrm{~cm}^{-2}$ and an excitation temperature of $220 \pm 20 \mathrm{~K}$ is required. These lines have an optical depth, $\tau$, of $\sim 0.7$. The $\mathrm{CH}_{3} \mathrm{OH}$ emission shown in Fig. 8 meanwhile indicates that the lines may be optically thick towards the protostellar position. However, the spectra extracted over the entire emitting region have line profiles with $\tau<1$, which suggests that the optically thin approximation is overall appropriate. The excitation temperature derived from the spectra is consistent with what is found on 5 au scales when using the previously estimated dust temperature 
of $30 \mathrm{~K}$ at 600 au (Chandler \& Sargent 1993), and assuming that its dependence on the distance from the protostar follows a power-law with index -0.4 (Yen et al. 2015; Shirley et al. 2000).

\section{Discussion}

\subsection{Mass of the circum-protostellar emission}

The circumstellar material traced by the continuum in our longbaseline observations is well-fitted by a 2D Gaussian with a FWHM of $\sim 7 \mathrm{au}$, and $S_{1.3 \mathrm{~mm}}=28 \pm 0.2 \mathrm{mJy}$. The continuum emission is therefore barely spatially resolved. The mass of the emitting region is estimated from

$M_{1.3 \mathrm{~mm}}=\frac{S_{1.3 \mathrm{~mm}} d^{2}}{\kappa_{1.3 \mathrm{~mm}} B\left(T_{\text {dust }}\right)}$,

where $\kappa_{1.3 \mathrm{~mm}}$ is the dust mass opacity at $1.3 \mathrm{~mm}, d$ is the distance to $\mathrm{B} 335$, and $B\left(T_{\text {dust }}\right)$ is the Planck function at the temperature of the dust, $T_{\text {dust }}$. To enable comparison with earlier results, we use a total mass opacity of $0.014 \mathrm{~cm}^{2} \mathrm{~g}^{-1}$ (Yen et al. 2015; Chandler \& Sargent 1993). This value is similar to tabulated values for MRN grains (Mathis et al. 1977) with thin ice mantles at high densities and a standard gas-todust mass ratio (Ossenkopf \& Henning 1994), but is slightly lower than what is generally assumed for Class I and II disks (e.g. Ricci et al. 2010; Harsono et al. 2018). Assuming that the gas temperature in the methanol emitting region is a good proxy for the dust temperature in the continuum emitting region (200 K; see Sect. 3.3), the resulting circumstellar mass within 7 au is $M_{\text {dust }}=3 \times 10^{-4} M_{\odot}$. This mass is comparable to the $7.5 \times 10^{-4} M_{\odot}$ inferred within a 25 au radius by Evans et al. (2015), and in the case of spherical symmetry, therefore, consistent with a power-law density distribution with index, $p=-2$ (Yen et al. 2015). The circumstellar mass is two orders of magnitude smaller than the $0.05-0.15 M_{\odot}$ (Yen et al. 2015; Evans et al. 2015) that has accumulated in the central zone, as inferred from models of inside-out collapse (Shu 1977). Since the temperature gradients on even smaller scales cannot be measured with our current data, we note that the circumstellar mass could in fact be lower. For instance, by Eq. (1), increasing the dust temperature by a factor of two yields a factor of two lower mass.

\subsection{Infall and rotation on small scales}

Position-velocity cuts in the direction perpendicular to the outflow axis and across the protostellar position are presented together with the integrated emission $\left( \pm 5 \mathrm{~km} \mathrm{~s}^{-1}\right.$, w.r.t. thesystemic velocity) in Fig. 8. The FWHM of the emitting regions are estimated from 2D Gaussian fits to the emission ${ }^{6}$.

The PV diagram of ${ }^{13} \mathrm{CO}$ shows a pronounced bluered asymmetry; the blueshifted component is clearly visible while the redshifted components is nearly entirely absent. The observed blueshifted emission is consistent with infall towards a $0.05 M_{\odot}$ point-source (green dashed lines in Fig. 8), and which is substantially larger than the estimated mass of the circumstellar region $\left(3 \times 10^{-4} M_{\odot}\right)$, but comparable to the mass estimated from inside-out collapse $\left(0.05-0.15 M_{\odot}\right.$; see Sect. 4.1). A blueshifted, higher velocity component $\left(\lesssim 5 \mathrm{~km} \mathrm{~s}^{-1}\right)$ is visible in the PV diagram, and its offset from free-fall and Keplerian rotation curves

\footnotetext{
6 Since the errors on the FWHM are lower than 1 au in all cases, the errors are not reported here.
}

suggest that it may be associated with the outflow. However, we find no significant signature of rotation in the ${ }^{13} \mathrm{CO}$ emission. The FWHM of the ${ }^{13} \mathrm{CO}$ emitting region is estimated at $22 \mathrm{au}$, that is, extended compared to the size of the beam.

The PV diagrams for the two $\mathrm{CH}_{3} \mathrm{OH}$ transitions exhibit a distinctly different morphology and kinematics relative to ${ }^{13} \mathrm{CO}$. The FWHM of the emitting $\mathrm{CH}_{3} \mathrm{OH}$ regions are estimated at 16 and 13 au for $v=0$ and $v=1$ lines, respectively, which is significantly smaller than the region probed by ${ }^{13} \mathrm{CO}$. The highest velocities observed in $\mathrm{CH}_{3} \mathrm{OH}$ are found at small separations from the protostar, which is consistent with an infalling, rotating flow inferred from previous observations (Yen et al. 2015). A rotation signature, consistent with solid-body rotation, is observed. However, the extent of the emission, plus the sensitivity limits on the higher velocity emission, do not allow us to significantly constrain the rotation profile. Given the current data, we can therefore not rule out Keplerian rotation around a $0.05 M_{\odot}$ protostar (red dashed lines in the right panels of Fig. 8).

That said, we examined the velocity gradient in the immediate vicinity of the protostar in detail, and derived the peak position of the emission as a function of velocity by fitting Gaussians to the flux in each channel in the PV diagram. The ability of a fit to reproduce the flux profile in each channel is evaluated using the coefficient of determination ("R squared"), where a value of 1 indicates that the variance is fully accounted for by the fit. We present only velocity channels where R squared is larger than 0.3 and then subsequently fit a straight line through the peak positions (a lower threshold does not affect the results) assuming that the source velocity is $8.3 \mathrm{~km} \mathrm{~s}^{-1}$ w.r.t. $v_{\text {LSR }}$. The errors on the estimated peak emission positions are included as parameters in the fit. As shown in the right panels of Fig. 8, we arrive at velocity gradients that are perpendicular to the outflow axis with values of $1.4 \pm 0.3$ and $0.9 \pm 0.5 \mathrm{~km} \mathrm{~s}^{-1} \mathrm{au}^{-1}$ for the $v=0$ and $v=1$ transitions of $\mathrm{CH}_{3} \mathrm{OH}$, respectively. These values can be compared to the velocity gradient of $0.27 \pm 0.03 \mathrm{~km} \mathrm{~s}^{-1} \mathrm{au}^{-1}$ derived from the $\mathrm{C}^{18} \mathrm{O}$ emission on scales $\sim 30$ au, as reported by Yen et al. (2015). These authors observed B335 at an order of magnitude coarser angular resolution than the current longbaseline observations. They are thus probing scales where any Keplerian rotation would be a factor of three smaller, while in the case of solid-body rotation, velocities would be a factor of ten higher. By combining the extent of the observed $\mathrm{CH}_{3} \mathrm{OH}$ emission and the derived velocity gradient, we estimate the specific angular momentum within $\sim 20$ au to be $\sim 25$ au km s${ }^{-1}$.

Due to the compact and clearly rotating nature of the methanol emission, and the fact that it rotates at a velocity that is a factor of three to four times higher than what was previously derived from $\mathrm{C}^{18} \mathrm{O}$ at larger scales, we suggest that the methanol emitting region is associated with an inner, rotating, and possibly disk-like structure.

In the case of $\mathrm{SO}_{2}$, using Gaussian fitting, we find the emission has a FWHM of $\simeq 10$ au, that is, even smaller than that of $\mathrm{CH}_{3} \mathrm{OH}$. We note that only a small amount of blueshifted emission is detected on the northern side of the source, and vice versa on the southern side; if the emission was predominantly tracing infall, we would expect to see blueshifted emission on the northern side of the PV diagram (and vice versa for the southern side). We believe the reason for this is that the rotational velocity component is dominant over the infalling velocity component in this region. It has recently been suggested that $\mathrm{SO}_{2}$ can, in fact, be a tracer of accretion shocks close to the protostar (Artur de la Villarmois et al. 2018). Unfortunately, although $\mathrm{SO}_{2}$ shows the most compact morphology of the species detected in these 
observations, we cannot, at present, draw any firm conclusions regarding its origin. What is clear, however, is that the observed gas is rotating on the very smallest scales.

\subsection{The outflow cavity walls}

The combined observations presented here recover most of the ${ }^{12} \mathrm{CO}$ emission in the line wings relative to single-dish observations (cf. Fig. 2). Thus, we claim that the majority of the ${ }^{12} \mathrm{CO}$ emission in B335 originates in the narrow arms of emission that follow the X-shaped cavity walls (Figs. 3 and 4). Although the width of these arms (up to $\sim 50 \mathrm{au}$ ) vary slightly along the cavity walls, the width is almost always larger than our beam size, and most of the cavity emission is resolved.

While the peak of the continuum emission is concentrated at the protostellar position in both long-baseline and combined data, only the combined data set reveals extended $1.3 \mathrm{~mm}$ emission (Figs. 4 and A.1) to the north and south. The most striking feature of the continuum (greyscale; Fig. A.1) is that it stretches $\sim 800$ au to the north (that is, greater than the extent of Fig. 4) and a few hundred au to the south. In addition, the map shows that the dust emission follows the cavity walls, especially to the south-west, in agreement with Maury et al. (2018). However, it is only prominent at separations from the outflow axis which are greater than for the ${ }^{12} \mathrm{CO}$ emission, and the apparent opening angle (when examining the brightest dust continuum emitting regions to the east) is slightly larger than the opening angle of the ${ }^{12} \mathrm{CO}$ emission. Although we cannot entirely refute that the dust is being carried by the outflow, the coincidence with the $\mathrm{X}$-shaped morphology of the ${ }^{12} \mathrm{CO}$, suggests that the wind is excavating a cavity and/or entraining dust from the envelope, rather than transporting it from the circum-protostellar region. Under the reasonable assumption that a significant fraction of the ${ }^{12} \mathrm{CO}$ emission in the cavity walls (which extends for $\gtrsim 2000 \mathrm{au}$ ) is caused by mechanical interaction between the outflow and the envelope, the cavity wall emission provides an estimate on the lateral scales over which entrainment takes place.

That said, while the edge of the continuum emission is in close proximity of the ${ }^{12} \mathrm{CO}$ emission, it is possible that some of the emission observed in the north and south is actually tracing the infalling surrounding envelope. In any case, to our knowledge, B335 is the only protostellar example where dust continuum is detected towards an outflow cavity wall.

\subsection{Outflow rotation and cavity expansion}

Since Keplerian rotation has not been detected towards B335 on scales larger than $10 \mathrm{au}$, it is interesting to instead search the long-baseline data for signs of outflow rotation. Unfortunately, the ${ }^{12} \mathrm{CO}$ line profiles are dominated by infall and outflow motions and we find no significant evidence of rotation in the outflow. As mentioned in Sect. 3.2, however, $\mathrm{CH}_{3} \mathrm{OH}$ and $\mathrm{SO}_{2}$ do show clear evidence for rotation within $\sim 16$ au (Fig. 8). This emission originates in the central region, where it appears that material is infalling and rotating simultaneously. Even so, the velocity gradients measured from the $\mathrm{CH}_{3} \mathrm{OH}$ and $\mathrm{SO}_{2}$ lines are consistent with one another, and thus one might expect that the outflow should rotate in the same sense.

A ${ }^{12} \mathrm{CO}$ moment 1 map (Fig. A.3) and line profiles at different positions (Fig. 4), meanwhile, do not show any significant difference in the velocities between northern and southern outflow cavity walls. In the redshifted outflow, however, there is a blueshifted component at small separations $(\$ 30 \mathrm{au})$ south-west of the protostar (Fig. 3), consistent with the inferred rotation seen in $\mathrm{SO}_{2}$ and $\mathrm{CH}_{3} \mathrm{OH}$. Likewise, a redshifted component is observed in the blueshifted outflow on comparable scales, but this component is seen along the jet axis and not the northwestern side as would be expected if this component was due to rotation in the outflow.

Attributing the inner features to rotation is in any case difficult since the outflow is nearly in the plane of the sky, and expansion of the outflow, deviations of the outflow axis from axisymmetry, or internal shocks (e.g. Fendt 2011) can produce similar features. Hints of expansion are indeed seen in the lowermost PV diagram of Fig. 9, where an extended U-shaped structure is observed at blueshifted velocities. Although this emission structure barely exceeds the $3 \sigma$ threshold, it is clear that the velocity is increasing towards the outflow axis. A similar feature is found on the redshifted side at slightly lower $\mathrm{S} / \mathrm{N}$, and lower velocities. While an onion-layered velocity structure (whereby velocities close to the outflow axis are high and then fall off with distance) is expected for a rotating, magneticallylaunched wind (e.g. Bacciotti et al. 2002; Pudritz et al. 2006), the symmetry of the PV diagram w.r.t. the zero position offset in the lowermost panel of Fig. 9 is not consistent with this picture. It is, however, consistent with expanding cavity walls.

The rotation and expansion velocity at different offsets from the protostar can be estimated using the method presented in Lee et al. (2018), assuming that only two velocity components are present. While this is an oversimplification of reality, assuming the outflow velocity does not change significantly over the considered distances, it does provide a useful method to deduce any trends in rotation and expansion.

We fit 2D Gaussians to several PV diagrams with cuts perpendicular to the outflow axis but with different offsets. One example of such a fit (at zero offset from the continuum peak) is presented in the uppermost panel of Fig. 9). We only consider pixels where the emission is above $3 \sigma$, and we purposefully mask out velocities between 7.3 and $9.3 \mathrm{~km} \mathrm{~s}^{-1}$, that is, where emission from the foreground is expected to be significant ${ }^{7}$. With this approach, the rotation velocity is given by the $1 \sigma$ velocity at maximum extension with respect to the central velocity of a Gaussian fit to the emission. Meanwhile, the expansion velocity is given by the difference between the velocity at the zero position offset and the central velocity of the Gaussian fit.

Figure 10 shows the results of the fitting procedure, but clear evidence for rotation in the outflow cannot be inferred from this analysis except at $\lesssim 20$ au separation from the protostar in the redshifted outflow, where the values are on the $1 \mathrm{~km} \mathrm{~s}^{-1}$ level. Since any rotational motions of the ${ }^{12} \mathrm{CO}$ outflow are apparently at a minor level, and given that outflows, if magneto-centrifugally-powered, are expected to rotate (Blandford \& Payne 1982; Pudritz \& Norman 1983), it is reasonable to assume that a large fraction of the $\mathrm{CO}$ emitting gas is instead material that is being entrained by the outflow.

In addition, the inferred expansion velocities in the outflow, $\sim(2-4) \mathrm{km} \mathrm{s}^{-1}$, are at least an order of magnitude lower than what is needed to explain the observed width of the outflow given the forward velocities previously inferred from proper motion of the $\mathrm{HH}$ objects, assuming its width is due entirely to expansion.

Considering these results, we suggest that what we predominantly are seeing in ${ }^{12} \mathrm{CO}$ is the excavation of a cavity and entrainment of material, rather than the wind itself.

\footnotetext{
We note, however, that including velocities from $7.3-9.3 \mathrm{~km} \mathrm{~s}^{-1}$ in our analysis does not affect our results.
} 

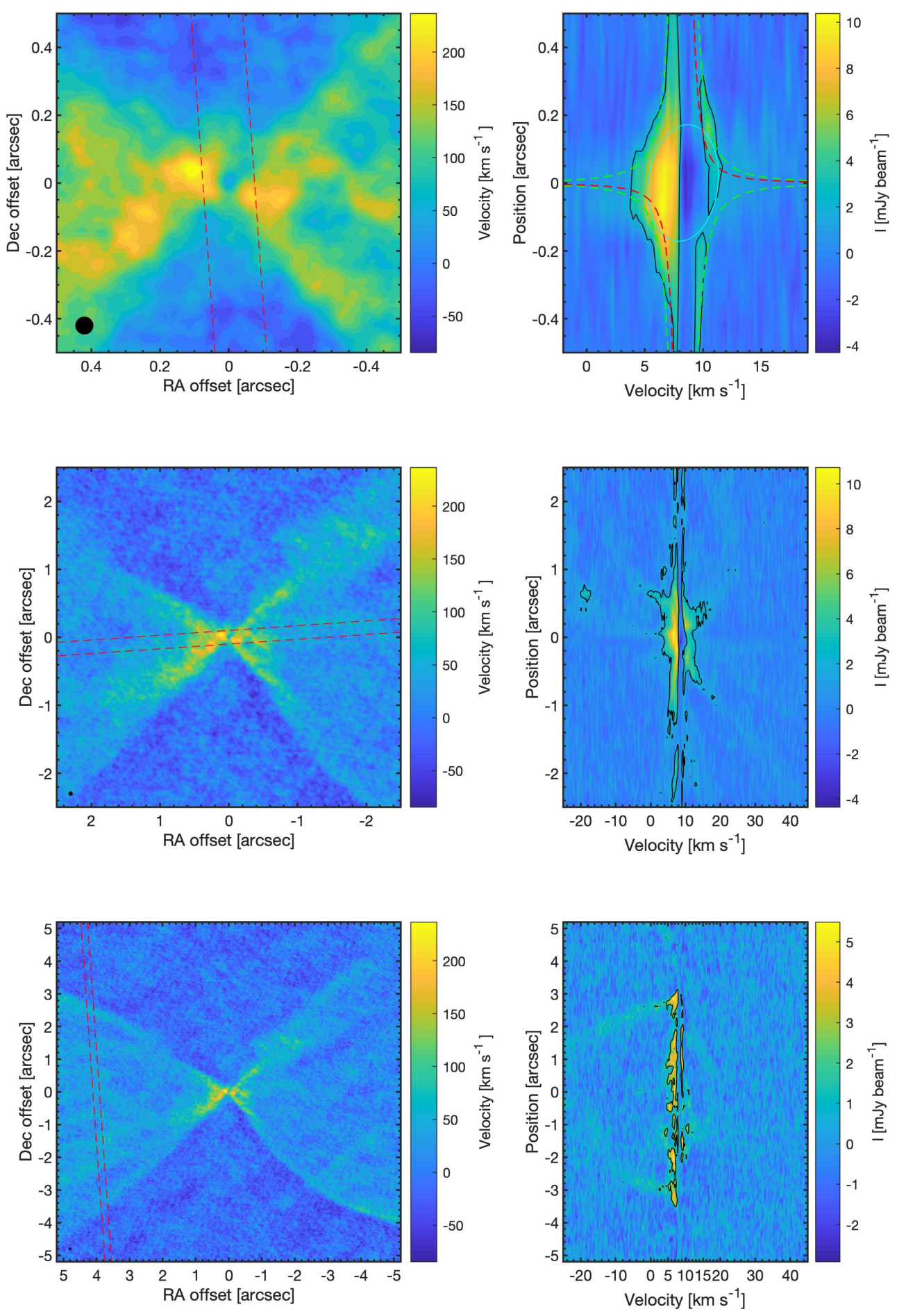

Fig. 9. ${ }^{12} \mathrm{CO}$ integrated emission and PV diagrams. Left panels: moment 0 maps of the ${ }^{12} \mathrm{CO}$ emission (within $\pm 5 \mathrm{~km} \mathrm{~s}^{-1}$ from the systemic velocity) overlaid with the orientation and width of each PV cut. The maps were convolved with a 2D Gaussian to 5 au resolution to improve the S/N. Right panels: PV diagrams for ${ }^{12} \mathrm{CO}$ along the different cuts shown in the left panels. Contours are at $3 \sigma$. In the upper right panel, green and red dashed lines denote pure free-fall and Keplerian rotational velocities towards/around a $0.05 M_{\odot}$ protostar, respectively. An example of a 2D Gaussian fit (see Sect. 4.4) to the emission is indicated by the cyan ellipse. Note the change in scale from top to bottom rows.

\subsection{Launching of the wind}

The idea that protostellar jets are launched magnetocentrifugally from disks is now widely accepted, although several unresolved issues remain (see, e.g. Frank et al. 2014). In our long-baseline data, the intersection point of the X-shaped morphology of the ${ }^{12} \mathrm{CO}$ emission is consistent with small outflow launching radii. This is supported by the scale of the detected $\mathrm{SiO}$ emission that we associate with a central jet. The south-western and north-eastern cavity walls exhibit little curvature, while the other two cavity walls show no evidence for curvature at all. In the case of a magnetically-launched outflow, not only is the same mechanism responsible for the transfer of both kinetic energy and angular momentum into the wind, the outflow rotational and forward velocity components should also be closely linked (e.g. Ferreira et al. 2006; Ramsey \& Clarke 2019). This remains true whether the underlying mechanism is a disk wind and/or an X-wind (Shu et al. 1994).

The middle panels of Fig. 9 presents the PV diagram along the outflow for the combined ${ }^{12} \mathrm{CO}$ data set, and reveals some interesting features. First, at small positional offsets (within $100 \mathrm{au}$ from the protostar), a single, significant, isolated high velocity blueshifted component can be seen at velocities reaching $\sim 30 \mathrm{~km} \mathrm{~s}^{-1}$ w.r.t. the systemic velocity. In addition, both 


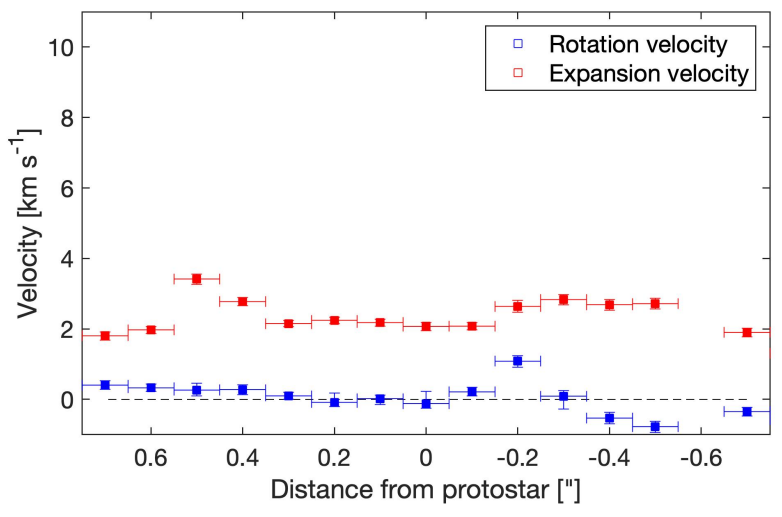

Fig. 10. Rotation and expansion velocities of the outflow derived from $\mathrm{PV}$ cuts of the ${ }^{12} \mathrm{CO}$ emission across the outflow axis with different separations from the protostar. Error bars are given by the uncertainty in the 2D Gaussian fits and the width of the PV cut.

blue- and redshifted components show marginally detected emission which extends out to $\sim 20 \mathrm{~km} \mathrm{~s}^{-1}$ and offsets of $\sim 100 \mathrm{au}$. These velocity components are likely associated with a small scale jet that is expected along the central axis of the outflow. Second, in the redshifted outflow, a feature is visible at offsets of up to $50 \mathrm{au}$, corresponding to a marginally detected high velocity feature visible in Fig. 4 (red point/spectrum).

Although we do not detect any rotation in the outflow itself, the detection of rotation in $\mathrm{CH}_{3} \mathrm{OH}$ on the smallest scales allows us to put an upper limit on the rotational velocity component of the order of $1 \mathrm{~km} \mathrm{~s}^{-1}$. One can thus estimate the launching radius if the protostellar mass is known. Following Anderson et al. (2003), we use a velocity of $1.0 \mathrm{~km} \mathrm{~s}^{-1}$ and a maximum line-of-sight velocity of $\simeq 30 \mathrm{~km} \mathrm{~s}^{-1}$, estimated from the aforementioned structures seen in the ${ }^{12} \mathrm{CO}$ PV cuts taken along the outflow at $\sim 60$ au separation from the central source (where the velocities are higher then the local escape velocity). The width of the outflow in this region is of the order $80 \mathrm{au}$. We adopt an inclination angle $10^{\circ}$ (Hirano et al. 1988) with respect to the plane of the sky, yielding a maximum ${ }^{12} \mathrm{CO}$ velocity of $\sim 170 \mathrm{~km} \mathrm{~s}^{-1}$ in the outflow direction. Applying Eq. (4) of Anderson et al. (2003), we thus estimate an upper limit for the launching radius of the high-velocity CO gas of the order of $0.07 \mathrm{au}$. Decreasing the rotation velocity by a factor of three implies a factor of two smaller launching radius $\left(r_{0} \propto v_{\phi}^{2 / 3}\right)$. We also note that a smaller inclination angle w.r.t. the plane of the sky implies even smaller launching radii.

The lack of a clear rotation signature in the ${ }^{12} \mathrm{CO}$ outflow prevents us from calculating the specific angular momentum in the regions of high velocity. We can, however, provide an upper limit of 40 au $\mathrm{km} \mathrm{s}^{-1}$ (maximum rotational velocity is estimated at $1 \mathrm{~km} \mathrm{~s}^{-1}$ within 30 au from the protostar, where the width of the outflow is less than $40 \mathrm{au}$ ), which is consistent with the angular momentum derived from the observed rotation in methanol, viz. $25 \mathrm{au} \mathrm{km} \mathrm{s}^{-1}$ (Sect. 4.2).

\subsection{A potential recent ejection outburst}

One way to characterise the accretion history of protostars is to study how the local chemistry is affected by a short burst in accretion luminosity (e.g. Bjerkeli et al. 2016a). However, a disadvantage of this method is that it is only sensitive to relatively long time scales (e.g. 100-1000 yr; Jørgensen et al. 2013). An alternative approach is to take advantage of the fact that increased accretion should also lead to enhanced outflow activity (e.g. Raga et al. 1990). This method has previously been used on large spatial scales (e.g. Plunkett et al. 2015) to constrain the accretion history of protostars. With our current data, we can now apply the same analysis to very small spatial scales, and hence, on small temporal scales.

Emission from ${ }^{12} \mathrm{CO}$ is clearly detected at $-30 \mathrm{~km} \mathrm{~s}^{-1}$ w.r.t. systemic velocity $\left(\sim 170 \mathrm{~km} \mathrm{~s}^{-1}\right.$ assuming an inclination of $\left.10^{\circ}\right)$ and $\sim 60$ au separation in the blueshifted outflow (Fig. 9; middle). The cause of this emission is not readily apparent from our data. It could be the continous, molecular component of a jet which is propagating along the outflow axis. This is not likely, however, since it is then not easy to explain why the emission is so spatially confined, and why it is only detected at an offset of $\sim 60 \mathrm{au}$. Alternatively, it could be due to a wobbling/precessing jet and there is therefore a significant component that is not confined to the plane of the sky. However, we find this scenario unlikely as well because of the overall X-shaped morphology of the outflow and the fact that the high velocity feature is located only at a distance ( $\sim 60 \mathrm{au})$ from the protostar. Instead, the explanation we find most plausible is that it is a molecular "bullet" associated with a recent accretion event and subsequent transient increase in ejection activity.

We interpret the features visible in the middle panels of Fig. 9 as evidence for such episodic ejection. For the feature at $-30 \mathrm{~km} \mathrm{~s}^{-1}$ w.r.t. systemic velocity and $\sim 60$ au separation in the blueshifted outflow, we can estimate its dynamical timescale: Deprojecting with $\cos (i)$ (assuming $i=10^{\circ}$; Hirano et al. 1988), the speed and distance of this feature from the continuum peak implies that ejection took place within the last few years (formally, $1.7 \mathrm{yr}$ ). This result is conspicuous. If outflow ejection was significantly enhanced only $2 \mathrm{yr}$ ago, it could be so that B335 very recently underwent a burst in accretion as well. This motivates a search of not only archival data, but also follow-up studies in the near future to monitor the accretion via episodic ejection events. It should be noted in this context, however, that if the inclination is closer to the plane of the sky (e.g. $3^{\circ}$; Stutz et al. 2008), that would imply even shorter dynamical time-scales.

\section{Summary and conclusions}

The isolated protostar B335 was observed using ALMA in its largest configuration $(\sim 16 \mathrm{~km}$ baselines). Not only are these the highest resolution observations of B335 to-date ( $\sim 3$ au resolution), by combining the long-baseline data with publicly available archival data, we are also able to probe large and small scales with high-fidelity simultaneously.

Dust continuum emission at $1.3 \mathrm{~mm}$ is seen in the longbaseline data towards the central position with a $F W H M=7$ au, slightly larger than the size of the synthesized beam of the observations. The dust mass of the central emission component is estimated to be $M_{\text {dust }}=3 \times 10^{-4} M_{\odot}$, consistent with earlier results.

Lines of $\mathrm{CH}_{3} \mathrm{OH}$ and $\mathrm{SO}_{2}$ are detected in the vicinity of the protostar $(F W H M<15 \mathrm{au})$ and show a rotational velocity gradient on the $1 \mathrm{~km} \mathrm{~s}^{-1} \mathrm{au}^{-1}$ level, corresponding to a specific angular momentum of $\sim 25$ au $\mathrm{km} \mathrm{s}^{-1}$. The PV diagrams for $\mathrm{CH}_{3} \mathrm{OH}$ and $\mathrm{SO}_{2}$ are consistent with Keplerian rotation around a protostellar mass of $0.05 M_{\odot}$. From LTE analysis of the $\mathrm{CH}_{3} \mathrm{OH}$ emitting region, we estimate a gas temperature of $220 \pm 20 \mathrm{~K}$ and a column density of $6.8 \pm 0.1 \times 10^{18} \mathrm{~cm}^{-2}$.

${ }^{12} \mathrm{CO}$ emission from the outflow shows an X-shaped morphology where most of the emission is detected in narrow arms along the cavity walls (Fig. 4). Comparison with single-dish observations suggests that the majority of the $\mathrm{CO}$ emitting gas 
is in the arms and is being entrained over a $\sim 50$ au thick region. $1.3 \mathrm{~mm}$ dust emission is also detected in the proximity of the presumed outflow cavity walls. Whether this dust is associated with the wind or excavated by the wind is, at present, not entirely clear. The coincidence between the continuum emission and the $\mathrm{X}$-shaped morphology of the ${ }^{12} \mathrm{CO}$ emission, combined with a lack of evidence for rotation along the "X", implies the dust is locally being excavated and/or entrained rather than having originated close to the protostar and transported in the outflow. Furthermore, the ${ }^{12} \mathrm{CO}$ emission does not reveal any obvious signs of rotation except on the very smallest scales where a blueshifted component is detected in the redshifted outflow to the south. Position-velocity cuts in the direction perpendicular to the outflow further suggest that, if the outflow is rotating, it is at a very low level. We also estimated the values of the expansion velocity (Fig. 10), but the values are not sufficient to explain the observed width of the outflow.

Within $\sim 60$ au of the protostar, episodic features moving at high velocities $\left(\sim 30 \mathrm{~km} \mathrm{~s}^{-1}\right)$ w.r.t. the systemic velocity are detected. The dynamical timescale of these knots is less than a few years. We estimate the launching radius of these episodic structures to be smaller than $0.1 \mathrm{au}$. In addition, $\mathrm{SiO}$ emission is observed to be elongated along the direction of the outflow and is likely associated with a jet, but does not show any clear signs of rotation on the scales probed here.

Taking all of the evidence together, and in particular the absence of a clear Keplerian disk on the smallest scales, suggests that B335 is either very young and/or disk growth is actively being inhibited by magnetic braking.

Acknowledgements. We would like to thank Neal Evans for a throrough referee report that greatly helped improve the quality of this paper. This paper makes use of the following ALMA projects data: 2013.1.00879.S, 2016.1.01552.S, and 2017.1.00288.S. ALMA is a partnership of ESO (representing its member states), NSF (USA) and NINS (Japan), together with NRC (Canada), MOST and ASIAA (Taiwan), and KASI (Republic of Korea), in cooperation with the Republic of Chile. The Joint ALMA Observatory is operated by ESO, AUI/NRAO and NAOJ. We acknowledge support from the Nordic ALMA Regional Centre (ARC) node based at Onsala Space Observatory. This paper also makes use of data acquired with the Atacama Pathfinder EXperiment (APEX) telescope. APEX is a collaboration between the Max Planck Institute for Radio Astronomy, the European Southern Observatory, and the Onsala Space Observatory. Swedish observations on APEX and the Nordic ARC node are supported through Swedish Research Council grant No. 2017-00648. P.B. acknowledges the support of the Swedish Research Council (VR) through contracts 2013-00472 and 2017-04924. The computations were performed on resources at Chalmers Centre for Computational Science and Engineering (C3SE), provided by the Swedish National Infrastructure for Computing (SNIC). Part of this work was supported by the German Deutsche Forschungsgemeinschaft, DFG project number Ts 17/2-1. JPR was supported, in part, by the Virginia Initiative on Cosmic Origins (VICO) and, in part, by the National Science Foundation (NSF) under grant nos. AST-1910106 and AST-1910675. The research of LEK is supported by a research grant (19127) from VILLUM FONDEN. J.K.J. is supported by the European Research Council (ERC) under the European Union's Horizon 2020 research and innovation programme through ERC Consolidator Grant "S4F" (grant agreement No 646908). Research at Centre for Star and Planet Formation is supported by the Danish National Research Foundation (DNRF97).

\section{References}

Alves, F. O., Girart, J. M., Caselli, P., et al. 2017, A\&A, 603, L3

Anderson, J. M., Li, Z.-Y., Krasnopolsky, R., \& Blandford, R. D. 2003, ApJ, 590, L107

Andre, P., Ward-Thompson, D., \& Barsony, M. 2000, Protostars and Planets IV (Tucson: University of Arizona Press), 59

Artur de la Villarmois, E., Kristensen, L. E., Jørgensen, J. K., et al. 2018, A\&A, 614, A26

Bacciotti, F., Ray, T. P., Mundt, R., Eislöffel, J., \& Solf, J. 2002, ApJ, 576, 222

Bjerkeli, P., Jørgensen, J. K., Bergin, E. A., et al. 2016a, A\&A, 595, A39

Bjerkeli, P., van der Wiel, M. H. D., Harsono, D., Ramsey, J. P., \& Jørgensen, J. K. 2016b, Nature, 540, 406
Blandford, R. D., \& Payne, D. G. 1982, MNRAS, 199, 883

Chandler, C. J., \& Sargent, A. I. 1993, ApJ, 414, L29

Endres, C. P., Schlemmer, S., Schilke, P., Stutzki, J., \& Müller, H. S. P. 2016, J. Mol. Spectr., 327, 95

Evans, II, N. J., Lee, J.-E., Rawlings, J. M. C., \& Choi, M. 2005, ApJ, 626, 919

Evans, II, N. J., Di Francesco, J., Lee, J.-E., et al. 2015, ApJ, 814, 22

Fendt, C. 2011, ApJ, 737, 43

Ferreira, J., Dougados, C., \& Cabrit, S. 2006, A\&A, 453, 785

Frank, A., Ray, T. P., Cabrit, S., et al. 2014, Protostars and Planets VI (Tucson: University of Arizona Press), 451

Gålfalk, M., \& Olofsson, G. 2007, A\&A, 475, 281

Harsono, D., Bjerkeli, P., van der Wiel, M. H. D., et al. 2018, Nat. Astron., 2, 646

Herbst, E., \& van Dishoeck, E. F. 2009, ARA\&A, 47, 427

Hirano, N., Kameya, O., Nakayama, M., \& Takakubo, K. 1988, ApJ, 327, L69

Högbom, J. A. 1974, A\&AS, 15, 417

Imai, M., Sakai, N., Oya, Y., et al. 2016, ApJ, 830, L37

Imai, M., Oya, Y., Sakai, N., et al. 2019, ApJ, 873, L21

Jørgensen, J. K., Bourke, T. L., Myers, P. C., et al. 2007, ApJ, 659, 479

Jørgensen, J. K., Visser, R., Sakai, N., et al. 2013, ApJ, 779, L22

Kristensen, L. E., van Dishoeck, E. F., Bergin, E. A., et al. 2012, A\&A, 542, A8

Lee, C.-F., Li, Z.-Y., Codella, C., et al. 2018, ApJ, 856, 14

Li, Z.-Y., Krasnopolsky, R., \& Shang, H. 2011, ApJ, 738, 180

Li, Z.-Y., Banerjee, R., Pudritz, R. E., et al. 2014a, Protostars and Planets VI (Tucson: University of Arizona Press), 173

Li, Z.-Y., Krasnopolsky, R., Shang, H., \& Zhao, B. 2014b, ApJ, 793, 130

Lindberg, J. E., Jørgensen, J. K., Brinch, C., et al. 2014, A\&A, 566, A74

Lynden-Bell, D. 1996, MNRAS, 279, 389

Machida, M. N., Inutsuka, S.-i., \& Matsumoto, T. 2014, MNRAS, 438, 2278

Mathis, J. S., Rumpl, W., \& Nordsieck, K. H. 1977, ApJ, 217, 425

Maury, A. J., Girart, J. M., Zhang, Q., et al. 2018, MNRAS, 477, 2760

McMullin, J. P., Waters, B., Schiebel, D., Young, W., \& Golap, K. 2007, in Astronomical Data Analysis Software and Systems XVI, eds. R. A. Shaw, F. Hill, \& D. J. Bell, ASP Conf. Ser., 376, 127

Mottram, J. C., Kristensen, L. E., van Dishoeck, E. F., et al. 2014, A\&A, 572, A21

Müller, H. S. P., Thorwirth, S., Roth, D. A., \& Winnewisser, G. 2001, A\&A, 370, L49

Müller, H. S. P., Schlöder, F., Stutzki, J., \& Winnewisser, G. 2005, J. Mol. Struct., 742, 215

Murillo, N. M., Lai, S.-P., Bruderer, S., Harsono, D., \& van Dishoeck, E. F. 2013, A\&A, 560, A103

Ohashi, N., Saigo, K., Aso, Y., et al. 2014, ApJ, 796, 131

Olofsson, S., \& Olofsson, G. 2009, A\&A, 498, 455

Ossenkopf, V., \& Henning, T. 1994, A\&A, 291, 943

Plunkett, A. L., Arce, H. G., Mardones, D., et al. 2015, Nature, 527, 70

Pudritz, R. E., \& Norman, C. A. 1983, ApJ, 274, 677

Pudritz, R. E., Rogers, C. S., \& Ouyed, R. 2006, MNRAS, 365, 1131

Raga, A. C., Binette, L., Canto, J., \& Calvet, N. 1990, ApJ, 364, 601

Ramsey, J. P., \& Clarke, D. A. 2019, MNRAS, 484, 2364

Reipurth, B., Heathcote, S., \& Vrba, F. 1992, A\&A, 256, 225

Ricci, L., Testi, L., Natta, A., et al. 2010, A\&A, 512, A15

Seifried, D., Banerjee, R., Pudritz, R. E., \& Klessen, R. S. 2013, MNRAS, 432, 3320

Shirley, Y. L., Evans, II, N. J., Rawlings, J. M. C., \& Gregersen, E. M. 2000, ApJS, 131, 249

Shu, F. H. 1977, ApJ, 214, 488

Shu, F. H., Adams, F. C., \& Lizano, S. 1987, ARA\&A, 25, 23

Shu, F., Najita, J., Ostriker, E., et al. 1994, ApJ, 429, 781

Snell, R. L., Loren, R. B., \& Plambeck, R. L. 1980, ApJ, 239, L17

Stutz, A. M., Rubin, M., Werner, M. W., et al. 2008, ApJ, 687, 389

Tobin, J. J., Hartmann, L., Chiang, H.-F., et al. 2012, Nature, 492, 83

Tomida, K., Tomisaka, K., Matsumoto, T., et al. 2013, ApJ, 763, 6

Tomida, K., Okuzumi, S., \& Machida, M. N. 2015, ApJ, 801, 117

van 't Hoff, M. L. R., Tobin, J. J., Trapman, L., et al. 2018, ApJ, 864, L23

Walsh, C., Loomis, R. A., Öberg, K. I., et al. 2016, ApJ, 823, L10

Wurster, J., \& Li, Z.-Y. 2018, Front. Astron. Space Sci., 5, 39

Yen, H.-W., Takakuwa, S., \& Ohashi, N. 2010, ApJ, 710, 1786

Yen, H.-W., Takakuwa, S., \& Ohashi, N. 2011, ApJ, 742, 57

Yen, H.-W., Takakuwa, S., Koch, P. M., et al. 2015, ApJ, 812, 129

Yildı, U. A., Kristensen, L. E., van Dishoeck, E. F., et al. 2015, A\&A, 576, A109

Zhao, B., Caselli, P., Li, Z.-Y., et al. 2016, MNRAS, 460, 2050 


\section{Appendix A: Supplementary material}

Figure A.1 presents continuum emission from Fig. 4 zoomed out by a factor of two. Figure A.2 shows the PV diagram for the
$\mathrm{SiO}(5-4)$ line detected in our long-baseline observations, but that was not presented in Fig. 8. Figure A.3 shows the ${ }^{12} \mathrm{CO}$ moment 1 map for the combined data set.

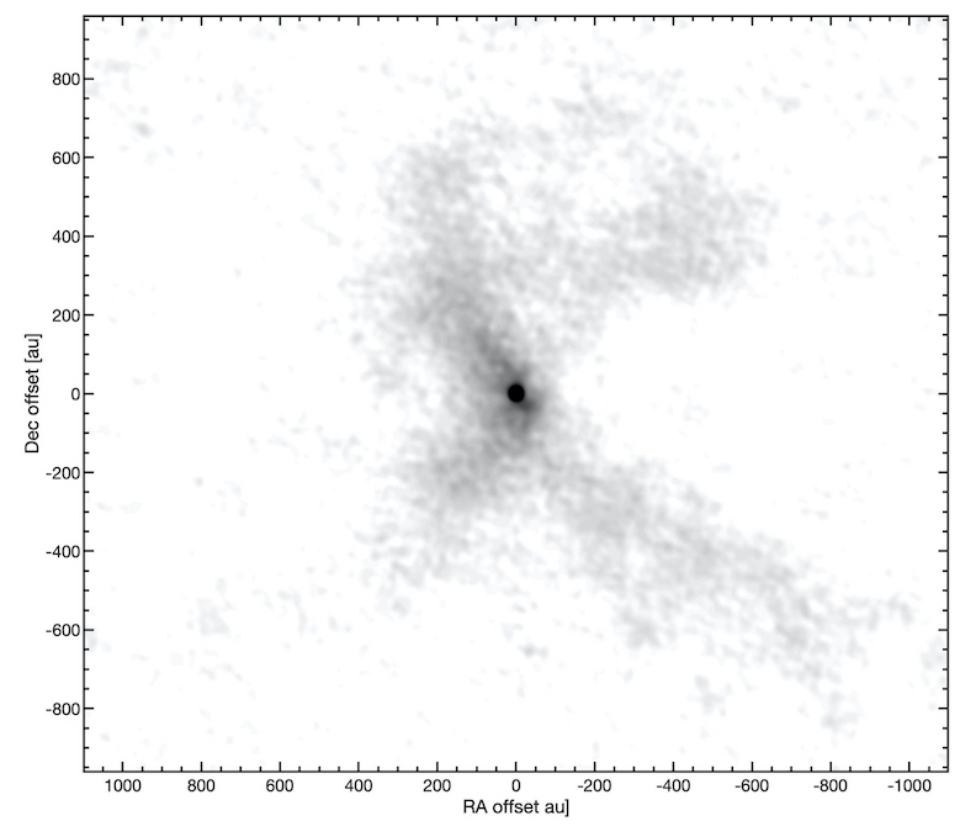

Fig. A.1. Same as Fig. 4, but continuum only and zoomed out by a factor of two.
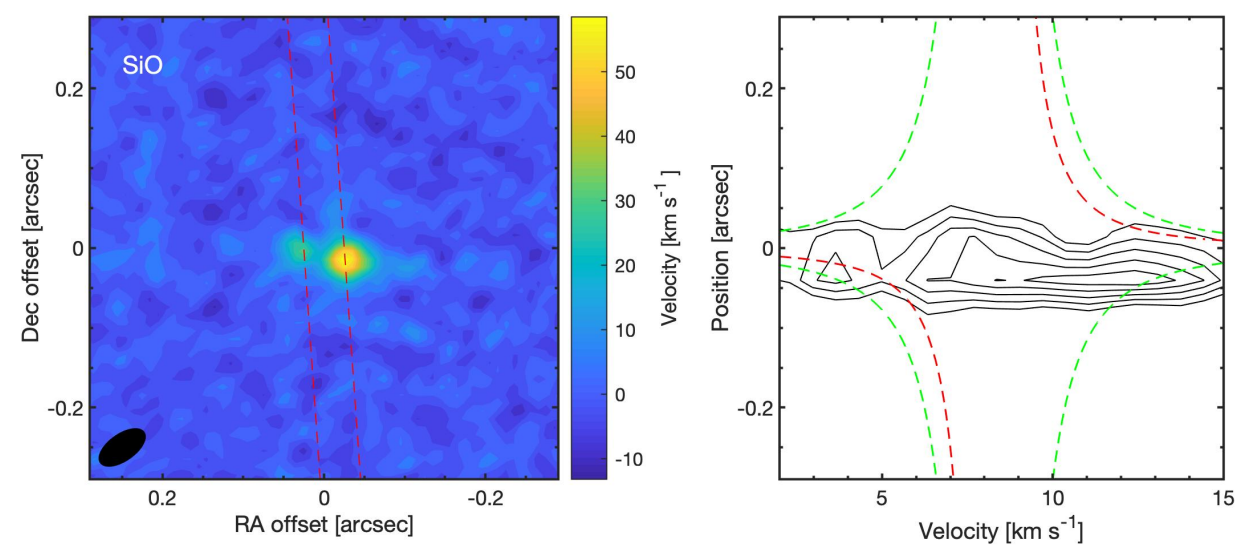

Fig. A.2. Same as Fig. 8, but for $\mathrm{SiO}(5-4)$. The $\mathrm{SiO}$ emission shows no clear evidence of rotation.

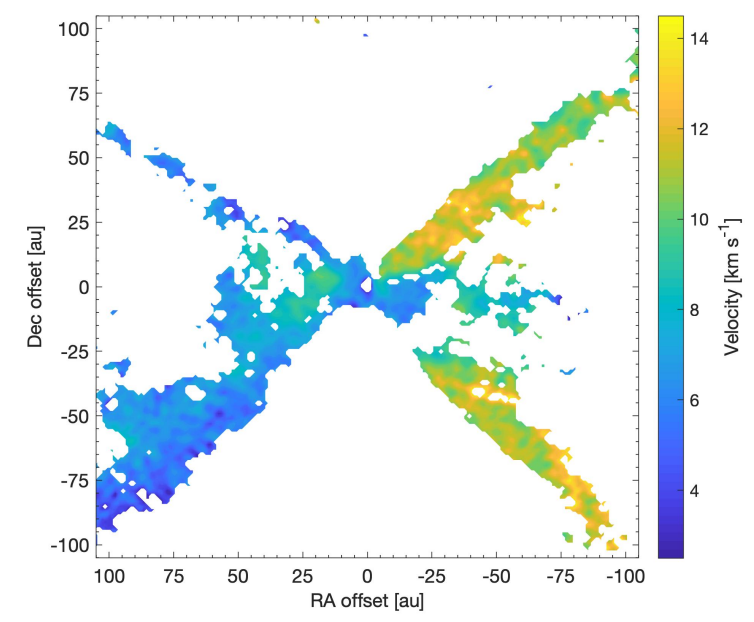

Fig. A.3. Moment 1 map for the combined ${ }^{12} \mathrm{CO}$ data. 